\title{
Sensitization of TRPA1 by PAR2 contributes to the sensation of inflammatory pain
}

\author{
Yi Dai, ${ }^{1,2}$ Shenglan Wang, ${ }^{1}$ Makoto Tominaga, ${ }^{3}$ Satoshi Yamamoto, ${ }^{4}$ Tetsuo Fukuoka, ${ }^{1}$ \\ Tomohiro Higashi, ${ }^{3}$ Kimiko Kobayashi, ${ }^{1}$ Koichi Obata, ${ }^{1}$ Hiroki Yamanaka, ${ }^{1}$ and Koichi Noguchi ${ }^{1}$

\begin{abstract}
1Department of Anatomy and Neuroscience, Hyogo College of Medicine, Hyogo, Japan. 2Department of Pharmacy, School of Pharmacy, Hyogo University of Health Sciences, Hyogo, Japan. ${ }^{3}$ Department of Physiological Sciences, Okazaki Institute for Integrative Bioscience,
\end{abstract} \\ National Institutes of Natural Sciences, Aichi, Japan. ${ }^{4}$ Department of Physiology, Hyogo College of Medicine, Hyogo, Japan.
}

\begin{abstract}
Proinflammatory agents trypsin and mast cell tryptase cleave and activate PAR2, which is expressed on sensory nerves to cause neurogenic inflammation. Transient receptor potential A1 (TRPA1) is an excitatory ion channel on primary sensory nerves of pain pathway. Here, we show that a functional interaction of PAR2 and TRPA1 in dorsal root ganglion (DRG) neurons could contribute to the sensation of inflammatory pain. Frequent colocalization of TRPA1 with PAR2 was found in rat DRG neurons. PAR2 activation increased the TRPA1 currents evoked by its agonists in HEK293 cells transfected with TRPA1, as well as DRG neurons. Application of phospholipase C (PLC) inhibitors or phosphatidylinositol-4,5-bisphosphate (PIP $)_{2}$ suppressed this potentiation. Decrease of plasma membrane PIP 2 levels through antibody sequestration or PLC-mediated hydrolysis mimicked the potentiating effects of PAR2 activation at the cellular level. Thus, the increased TRPA1 sensitivity may have been due to activation of PLC, which releases the inhibition of TRPA1 from plasma membrane PIP ${ }_{2}$. These results identify for the first time to our knowledge a sensitization mechanism of TRPA1 and a novel mechanism through which trypsin or tryptase released in response to tissue inflammation might trigger the sensation of pain by TRPA1 activation.
\end{abstract}

\section{Introduction}

The transient receptor potential (TRP) channels constitute a large and diverse family of channel proteins that are expressed in many tissues and cell types in both vertebrates and invertebrates. TRPA1 is a member of branch A of the TRP family of cation channels (1). It has been reported that TRPA1 forms channels activated by icilin, a chemical that induces a cooling sensation, and by temperatures less than or equal to $17^{\circ} \mathrm{C}(2)$. This channel was also reported to be activated by some pungent chemicals, such as horseradish, mustard oil, cinnamon oil, cannabinoids, and allicin (1-5). Mechanisms of activation of TRPA1 have been well studied recently $(2-4,6-8)$. However, the sensitization mechanism of this channel has not yet been elucidated. TRPA1 is expressed by a subset of small-sized dorsal root ganglion (DRG) or trigeminal ganglion neurons in neonatal and adult rats and mice $(4,9,10)$. Recent studies using knockout mice demonstrated that TRPA1 is an important component of the transduction machinery through which environmental irritants and endogenous proalgesic agents depolarize nociceptors to elicit inflammatory pain $(11,12)$. Thus, it is clear that this channel is one of the important transducers of noxious stimuli in the primary afferents.

PARs are a subfamily of G protein-coupled receptors (GPCRs) that share a unique mechanism of activation. Molecular cloning has identified 4 PARs, PAR-1-4 (13-17). Certain proteinases are

Nonstandard abbreviations used: AA, arachidonic acid; AITC, allyl isothiocyanate; B2R, bradykinin B2 receptor; DAG, diacylglycerol; DRG, dorsal root ganglion; EBSS, Earle's balanced salt solution; ET-18-OCH, 1 -O-octadecyl-2-O-methyl-sn-glycero-3phosphorylcholine; GF, GF109203X; GPCR, G protein-coupled receptor; IP3, inositol triphosphate; LR-NH2, H-Leu-Arg-Gly-Ile-Leu-Ser-NH2; NF200, neurofilament 200; OAG, 1-oleoyl-2-acetyl-sn-glycerol; $\mathrm{PIP}_{2}$, phosphatidylinositol-4,5-bisphosphate; PLC, phospholipase C; SL-NH2, H-Ser-Leu-Ile-Gly-Arg-Leu-NH2; TRP, transient receptor potential; TSA, tyramide signal amplification.

Conflict of interest: The authors have declared that no conflict of interest exists. Citation for this article: J. Clin. Invest. 117:1979-1987 (2007). doi:10.1172/JCI30951. known to cleave PARs within the extracellular amino terminus to expose a tethered ligand domain that binds and activates the cleaved receptors $(18,19)$. For 3 of the PARs (PAR-1, PAR2, and PAR-4), short synthetic peptides have been shown to activate the receptors without unmasking the tethered ligand (20). PARs are known to play important roles in the response to tissue injury, notably in the process of inflammation and repair (18). In particular, agonists of PAR2, tryptase and trypsin, released from different cell types including mast cells have widespread proinflammatory effects (21-24), in part via a neurogenic mechanism (25). PAR2 is expressed on a subset of primary sensory neurons, and PAR2 agonists stimulate release of substance $P$ and calcitonin gene-related peptide in peripheral tissues (25). Furthermore, it has been reported that PAR2 activation can sensitize adult rat DRG neurons in vitro and may contribute to the pathogenesis of pain in the pancreas, an organ in which inflammation results in activation of endogenous proteases such as trypsin (26). In addition to having neurogenic inflammatory effects, intraplantar injection of subinflammatory doses of PAR2 agonists in rats and mice is able to provoke prolonged thermal and mechanical hyperalgesia and elevate spinal Fos protein expression, indicating a direct role for PAR2 in pain transmission (27).

Recently, we reported that TRPV1 activity was sensitized by PAR2 in a PKC-dependent manner (28). Considering that a signaling pathway for PAR2 involves the activation of phospholipase $\mathrm{C}$ (PLC) via Gq/11 proteins, we hypothesized that a PAR2-mediated mechanism may also lead to TRPA1 sensitization in primary sensory neurons and hence contribute to the pathogenesis of inflammatory pain. In the present study, we observed significant coexpression of the TRPA1 with the PAR2 receptor in rat DRG neurons and found a functional interaction between PAR2 and TRPA1, both in a heterologous expression system and in rat DRG neurons, which was also confirmed at the behavioral level. 
A

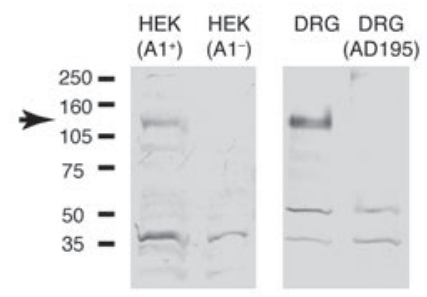

D
E

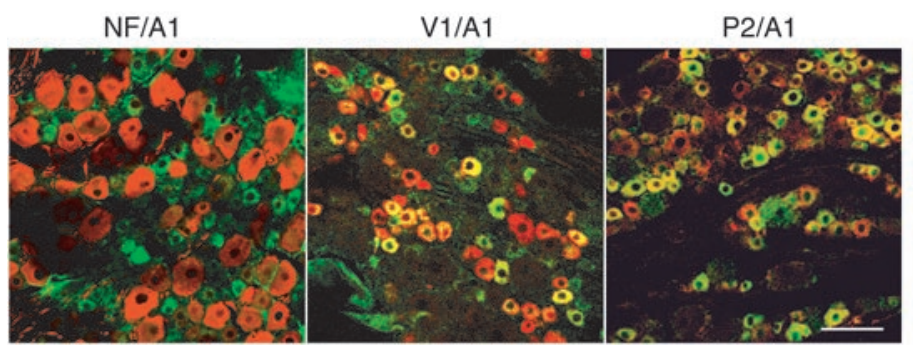

B

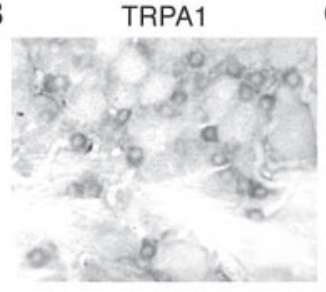

$\mathbf{F}$

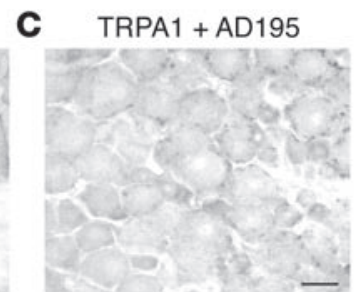

G

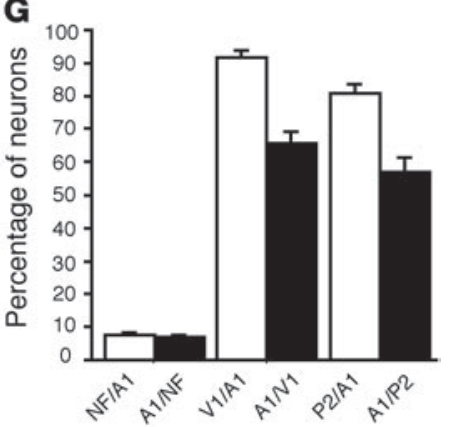

Figure 1

The antibody detected TRPA1 in rat DRG neurons. (A) The TRPA1 antibody recognized an expected band ( 128 kDa) in the Western blot from HEK cells heterogeneously expressing TRPA1 (left column) or from the rat DRG lysate (right column) but did not recognize the control HEK cells [HEK $\left(A 1^{-}\right)$] or DRG lysate pretreated with an excess of the antigenic peptide AD195 [DRG (AD195)]. (B and C) TRPA1 detected by immunohistochemistry in rat DRG neurons. The photographs show that TRPA1 was expressed in small DRG neurons (B); the label was eliminated by incubation of the antibody with an excess of the antigenic peptide AD195 (C). (D) Double immunostaining of TRPA1 (green) and NF200 (red) showed the TRPA1 expression in unmyelinated neurons of the rat DRG. (E) Double immunostaining of TRPA1 (green) and TRPV1 (red) showed that almost all of the TRPA1 were colocalized with TRPV1 in the rat DRG neurons. (F) Double immunostaining of TRPA1 (green) and PAR2 (red) showed that a large population of TRPA1 was colocalized with PAR2 in the rat DRG neurons. Yellow indicates double labeling. (G) Percentages of colocalization of NF200/TRPA1, TRPV1/TRPA1, and PAR2/TRPA1 in the DRG neurons. V1, TRPV1; A1, TRPA1; NF, NF200; P2, PAR2. Scale bars: $100 \mu \mathrm{m}$.

\section{Results}

Coexpression of TRPA1 with PAR2 in DRG neurons. In order to determine the distribution pattern of the TRPA1 channel protein, we raised a polyclonal antibody against the C-terminal 16-aa residues of rat or mouse TRPA1. This antibody recognizes a predicted band $(128 \mathrm{kDa})$ in immunoblots of extracts derived from HEK cells transfected with mTRPA 1 cDNA but not from the untransfected HEK cells (Figure 1A, left). This antibody recognized the predicted band $(128 \mathrm{kDa})$ from rat DRG extract. Pretreatment with excess antigenic peptide AD195 completely eliminated the band (Figure 1A, right). We used this affinity-purified antiserum on tissue sections from the rat DRG and found that TRPA1 was expressed by $33.5 \% \pm 3.7 \%$ of rat DRG neurons. The labeled neurons were mainly small in size (Figure $1 \mathrm{~B}$ ), which is consistent with previous reports $(9,10)$. The label was completely eliminated by preincubation of the antibody with an excessive amount of antigenic peptide AD195 (Figure 1C) but not with a control peptide AD194 (data not shown). Next, we examined the colocalization of TRPA1 with some neuronal markers in rat DRG neurons. Double immunofluorescence indicated that only $7.3 \% \pm 0.5 \%$ of TRPA 1 positive neurons had neurofilament 200 (NF200), a marker of myelinated primary afferent fibers, and $6.4 \% \pm 0.7 \%$ NF200-positive neurons were stained for TRPA1 (Figure 1, D and G). Therefore, TRPA1 is preferentially expressed in small, unmyelinated DRG neurons. Further, $91.6 \% \pm 1.2 \%$ of TRPA1-positive cells were also labeled for TRPV1, which is believed to be expressed exclusively in nociceptive neurons (10), while $65.6 \% \pm 2.1 \%$ of TRPV1-labeled neurons coexpressed TRPA1 (Figure 1, E and G). These data are highly consistent with previous reports of TRPA1 expression $(9,10)$. Taken together, these findings showed that the TRPA1 antiserum specifically recognized TRPA1 in rat DRG neurons.

Using this antibody, we performed double immunohistochemistry for PAR2 and TRPA1. We detected PAR2 expression in many smallsized and some medium-sized DRG neurons. A substantial population $(80 \% \pm 2.8 \%)$ of TRPA1-positive neuron were also labeled for PAR2, and $56.1 \% \pm 5.0 \%$ of PAR2-expressing neurons were labeled with TRPA1 (Figure 1, F and G). These high percentages of coexpression indicated a possible functional interaction between TRPA 1 and PAR 2 in primary afferent neurons.

PAR2 agonists sensitize TRPA1 in a heterologous expression system. To further determine the interaction of the TRPA1 with PAR2, we examined the effects of a synthetic-selective active peptide for PAR2, H-Ser-Leu-Ile-Gly-Arg-Leu-NH2 (SL-NH2), on the allyl isothiocyanate-activated (AITC-activated) currents in HEK293 cells expressing hTRPA1 using a whole-cell patch clamp technique. Because PAR2 is expressed endogenously in HEK cells (29), PAR2 cDNA was not transfected into HEK cells in the present study. In the voltageclamp experiments, AITC $(100 \mu \mathrm{M})$ did not induce any significant current in untransfected HEK cells. The AITC-activated inward current in the hTRPA1-transfected HEK293 cells displayed an outward rectification and were sensitive to ruthenium red (Figure 2A). The current underwent strong tachyphylaxis, giving much smaller response on repeated applications of $100 \mu \mathrm{M}$ AITC (Figure 2B). However, after 1 minute pretreatment with $100 \mu \mathrm{M}$ SL-NH2 (the PAR2 activator), reapplication of AITC with the same dose produced much larger current responses than the first application, 


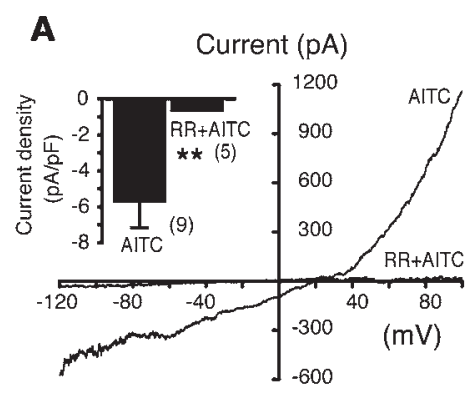

B AITC

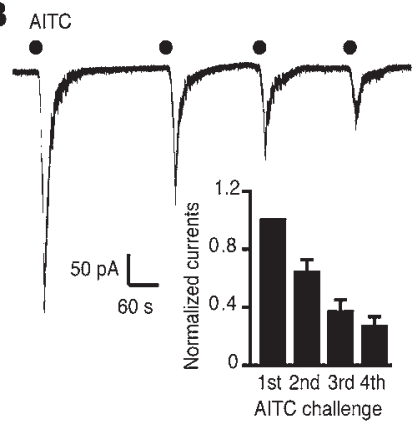

D

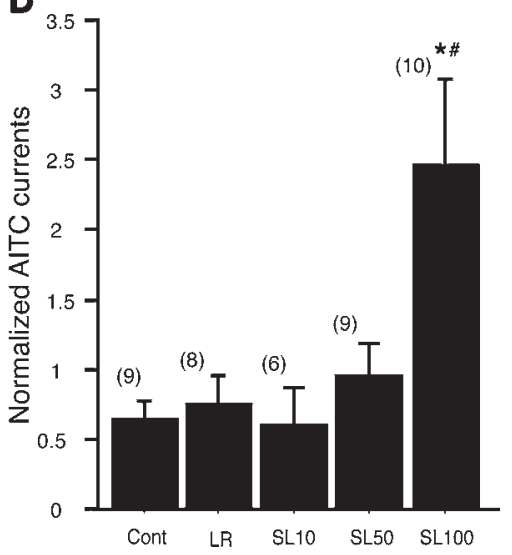

E

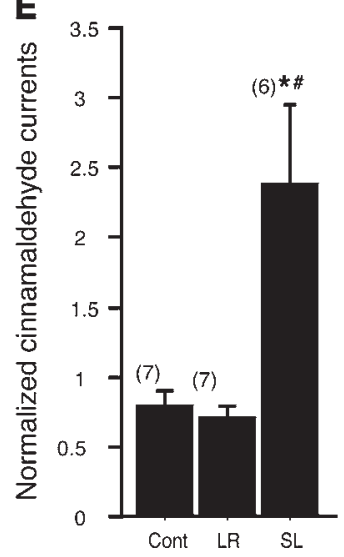

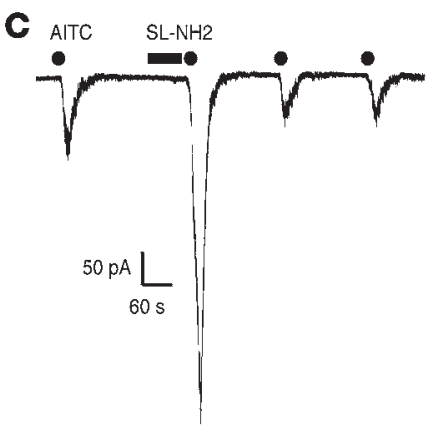

$\mathbf{F}$

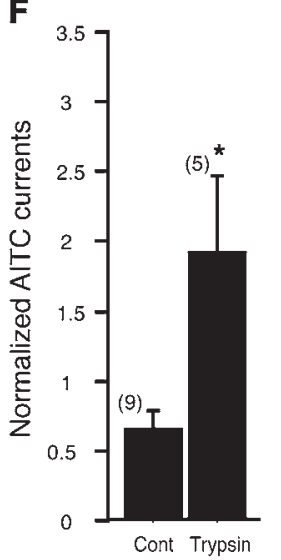

Figure 2

PAR2 agonists sensitize AITC- and cinnamaldehyde-activated currents in transfected HEK cells expressing hTRPA1 in a PLC-dependent manner. Cells were perfused with AITC $(100 \mu \mathrm{M})$ or cinnamaldehyde $(500 \mu \mathrm{M})$ solution for 20 seconds in all experiments. (A) Electrophysiological response of a representative transfected $\mathrm{HEK}$ cell to AITC in the absence or presence of ruthenium red (RR; $100 \mu \mathrm{M})$. Voltage ramps from -120 to +100 $\mathrm{mV}$ (340 ms) were applied. The inset graph shows AITC-activated current density in the absence or presence of ruthenium red $\left({ }^{\star \star} P<0.001\right)$. (B) AITC-activated inward currents underwent strong tachyphylaxis, giving much smaller responses on repeated applications of AITC. The inset graph shows normalized currents in each AITC challenge $(n=6)$. Currents were normalized to the currents evoked initially by AITC. (C) AITC-activated currents were sensitized after perfusion for 60 seconds with solution containing $100 \mu \mathrm{M}$ SL-NH2. However, this sensitization was not sustainable 180 seconds after SL-NH2 application. (D) SL-NH2-mediated potentiation of AITCactivated currents. AITC was reapplied 60 seconds after exposure to bath solution with or without SL-NH2 (SL; 10,50 , or $100 \mu \mathrm{M}$ ) or LR-NH2 (LR). Currents were normalized to values first induced by AITC application in the absence of SL-NH2 or LR-NH2. Cont, control group (preperfused with bath solution without SL-NH2 before reapplication of AITC). (E) Cinnamaldehyde-evoked TRPA1 currents were potentiated by SL-NH2 but not LR-NH2. (F) AITC-activated currents were potentiated by application of trypsin $(50 \mathrm{nM})$. Holding potential $\left(V_{\mathrm{h}}\right)$ was -60 $\mathrm{mV}$ in all experiments. Numbers in parentheses indicate cells tested. ${ }^{*} P<0.05$ versus control group; ${ }^{\#} P<0.05$ versus LR-NH2; unpaired Student's $t$ test.

which was not pretreated with SL-NH2 $(2.46 \pm 0.62$-fold change, $n=10$ for SL-NH2; $0.65 \pm 0.13$-fold change, $n=9$ for control; $P<0.05$ ) (Figure 2, C and D). Less potentiation or no potentiation was detected in cells pretreated with SL-NH2 in low concentrations $(10$ or $50 \mu \mathrm{M})$ or the reversed control peptide H-Leu-Arg-GlyIle-Leu-Ser-NH2 (LR-NH2), respectively $(0.60 \pm 0.26$-fold change, $n=5$ for $10 \mu \mathrm{M}, 0.95 \pm 0.23$-fold change, $n=9$ for $50 \mu \mathrm{M}$ of SL-NH2; $0.75 \pm 0.21$-fold change, $n=8$ for LR-NH2; $P>0.05$ for each versus control) (Figure 2D). Other electrophysiological properties of these AITC-evoked responses, including an outwardly rectifying current-voltage relationship and antagonist (ruthenium red) sensitivity, were unchanged by the presence of SL-NH2 (data not shown). A similar potentiating effect of SL-NH2 was observed on cinnamaldehyde-evoked activation of TRPA1 $(0.79 \pm 0.11$ fold change, $n=7$ for control; $0.70 \pm 0.08$-fold change, $n=7$ for $0.97 \pm 0.15$-fold change, $n=9$ for $\mathrm{U} 73122, P<0.05$ versus U73343, an inactive analog of U73122) (Figure 3A). In some experiments, application of U73122 alone induced small inward currents in HEK cells transfected with hTRPA1 independent of the application of any stimulus with an unknown mechanism. In addition to the inhibitory effect of PLC inhibitors, m-3M3FBS, a potent PLC activator, potentiated either the AITC- or the cinnamaldehydeactivated current $(3.17 \pm 0.94$-fold change, $n=15, P<0.05$ versus control for AITC; $2.15 \pm 0.33$-fold change, $n=7$ versus control for cinnamaldehyde) (Figure 3, B, C, and D). The current-voltage relationships for the currents activated by AITC after PLC activator ( $\mathrm{m}$-3M3FBS) application and those after application of the PAR2 agonist (SL-NH2) were nearly identical (data not shown).

We then examined whether the downstream effectors of PLC activation could sensitize TRPA1. One major downstream effect of 

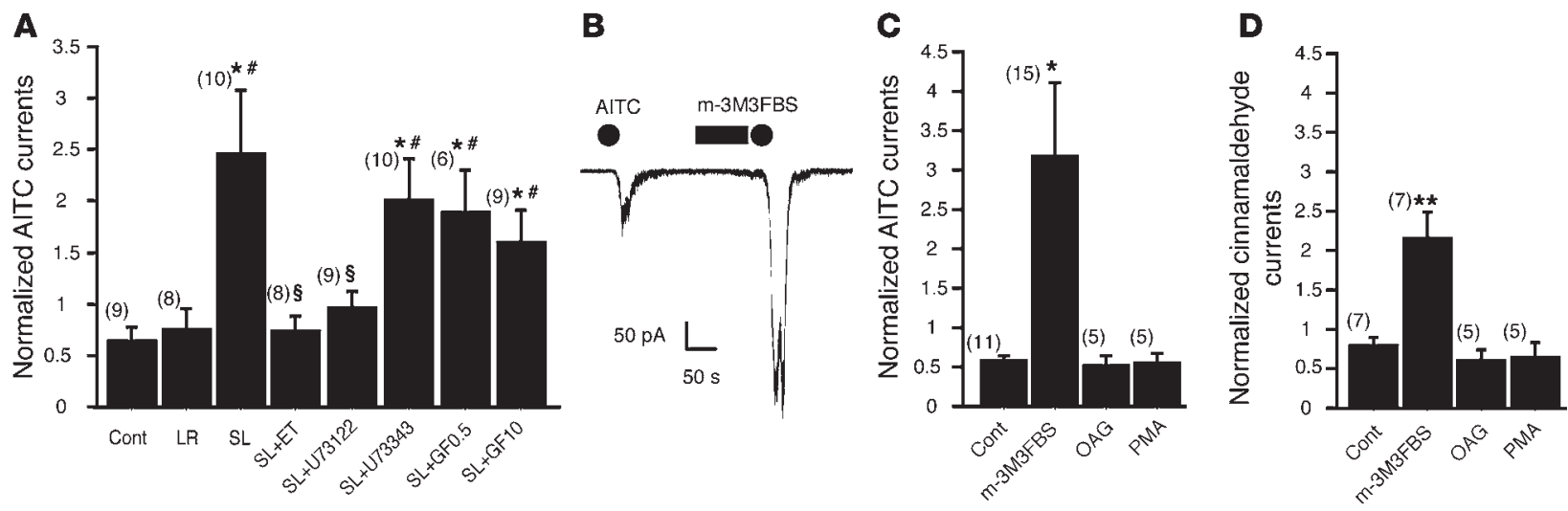

Figure 3

PLC activation, but not its downstream products, sensitized AITC- and cinnamaldehyde-activated currents in transfected HEK cells expressing hTRPA1. Cells were perfused with AITC or cinnamaldehyde solution for 20 seconds in all experiments. (A) SL-NH2-mediated (100 $\mu$ M) potentiation of AITC-activated currents is a PLC-dependent event. AITC $(100 \mu \mathrm{M})$ was reapplied 60 seconds after exposure to bath solution with or without SL-NH2 or LR-NH2. Currents were normalized to values first induced by AITC application in the absence of SL-NH2 or LR-NH2. In some experiments, the bath solution was perfused with either a PLC inhibitor - ET-18-OCH3 (ET; 2 mM) or U73122 (2 mM) - or a PKC inhibitor, GF $(0.5 \mathrm{mM}$ or $10 \mathrm{mM}) 120$ seconds before SL-NH2 reapplication. Numbers in parentheses indicate cells tested. ${ }^{*} P<0.05$ versus control group; $\# P<0.05$ versus LR-NH2; $\$ P<0.05$ versus SL-NH2; unpaired Student's $t$ test. (B) A representative trace of increase of AITC-activated current by a PLC activator, $\mathrm{m}-3 \mathrm{M} 3 F B S$. Cells were perfused for 90 seconds with solution containing $\mathrm{m}$-3M3FBS (10 $\mu \mathrm{M})$ before reapplication of the AITC. (C and D) Effects of PLC activation and its downstream products on the AITC- (30 $\mu \mathrm{M})$ (C) or cinnamaldehyde-activated (500 $\mu \mathrm{M})$ (D) current. Cells were perfused for 90 seconds with solution containing activators before reapplication of the AITC or cinnamaldehyde; $\mathrm{m}$-3M3FBS at $10 \mu \mathrm{M}$; OAG, a cell-permeable analog of DAG at $200 \mu \mathrm{M}$; and the PKC activator PMA at $1 \mu \mathrm{M}$ were used. Currents were normalized to the values evoked initially by AITC or cinnamaldehyde in the absence of the additives. Cells in the control group were perfused with bath solution without the additives before reapplication of agonists; ${ }^{*} P<0.05$, ${ }^{* *} P<0.005$ versus control group; unpaired Student's $t$ test. Note that OAG and PMA did not significantly potentiate the hTRPA 1 currents. Numbers in parentheses indicate cells tested. $V_{\mathrm{h}}$ was $-60 \mathrm{mV}$ in all experiments.

PLC activity is the generation of diacylglycerol (DAG). DAG can be converted to polyunsaturated fatty acid, such as arachidonic acid (AA), and induce PKC activation. We have recently reported that PAR2 activation caused potentiation or sensitization of TRPV1 through the PKC-dependent phosphorylation of TRPV1 (28). Therefore, we examined whether a similar signal transduction pathway was involved in the regulation of TRPA1 responses through PAR2. Unexpectedly, GF109203X (GF), a highly potent and specific PKC inhibitor, did not significantly inhibit the effect of SL-NH2, even at a very high concentration $(1.90 \pm 0.41$-fold change, $n=6$ for GF at $0.5 \mu \mathrm{M}, 1.61 \pm 0.32$-fold change, $n=9$ for GF at $10 \mu \mathrm{M}, P>0.05$ versus SL-NH2, $P<0.05$ versus LR-NH2) (Figure 3A). This result was supported by the lack of effect of PMA, a potent PKC activator, on TRPA1 potentiation ( $0.55 \pm 0.13$-fold change, $n=5$ for AITC; $0.64 \pm 0.19$-fold change, $n=5$ for cinnamaldehyde) (Figure 3, C and D). These data suggest that SL-NH2induced potentiation of TRPA1 responsiveness is independent of activation of PKC. We next tested whether 1-oleoyl-2-acetyl-sn-glycerol (OAG, a cell-permeable analog of DAG) could increase TRPA1 activity. OAG application did not significantly potentiate the AITC- or cinnamaldehyde-induced currents in hTRPA1-expressing HEK cells $(0.52 \pm 0.12$-fold change, $n=5$ for AITC; $0.61 \pm 0.14$-fold change, $n=5$ for cinnamaldehyde) (Figure 3, C and D).

Another major consequence of PLC activation is the release of calcium from intracellular stores. However, it is not a likely mechanism for the AITC-evoked current increase observed in our experiments, as cytosolic free $\mathrm{Ca}^{2+}$ was tightly chelated with the 5-mM EGTA included in the pipette solution. Taken together, the above results suggest that modulation of TRPA1 is dependent on PLC activation but does not involve its downstream products. Therefore, the mechanism of the SL-NH2-induced potentiation is likely the direct consequence of membrane phospholipid phosphatidylinositol-4,5-bisphosphate $\left(\mathrm{PIP}_{2}\right)$ hydrolysis by PLC activation.

GPCR-mediated potentiation of TRPA1 activity is mediated by $\mathrm{PIP}_{2}$ depletion. $\mathrm{PIP}_{2}$ has been shown to regulate the activity of a variety of ion channels (31). We thus asked whether PAR2-mediated sensitization of TRPA 1 channel activity was regulated by $\mathrm{PIP}_{2}$. When water-soluble $\mathrm{PIP}_{2}$ was diluted in the pipette solution to a final concentration of $10 \mu \mathrm{M}$, the effect of SL-NH2 was almost completely inhibited $(0.99 \pm 0.11$-fold change, $n=9, P<0.05$ versus SL-NH2) (Figure 4A). To further confirm the interaction of $\mathrm{PIP}_{2}$ with TRPA1, we intracellularly applied a PIP $_{2}$ monoclonal antibody or a $\mathrm{PIP}_{2}$ scavenger to sequester membrane $\mathrm{PIP}_{2}$. The $\mathrm{PIP}_{2}$ antibody application, but not the boiled, one mimicked the potentiation effect of SL-NH2 on AITC-activated hTRPA1 currents (1.05 \pm 0.17 -fold change, $n=5$ for control; $1.72 \pm 0.14$-fold change, $n=11$ for $\mathrm{PIP}_{2}$ antibody, $P<0.05$ versus control) (Figure $4, \mathrm{~B}$ and $\mathrm{C}$ ). Similarly, intracellular application of polylysine, a $\mathrm{PIP}_{2}$ scavenger, also potentiated the AITC-evoked currents $(0.65 \pm 0.13$-fold change, $n=9$ for control; $2.15 \pm 0.64$-fold change, $n=6$ for polylysine, $P<0.05$ versus control) (Figure 4D). These results suggest that endogenous $\mathrm{PIP}_{2}$ inhibits TRPA1 and that this repression can be alleviated by agents that activate PLC. Thus, SL-NH2-induced potentiation of TRPA1 responsiveness may develop through the membrane $\mathrm{PIP}_{2}$ hydrolysis by PLC activation. If inhibition of TRPA 1 activity by PIP $_{2}$ is a common phenomenon in mammalian cells, potentiation of TRPA1 current may occur by any other GPCR-mediated PLC activation. To answer this question, we transfected a bradykinin B2 receptor (B2R), which is a GPCR 
A

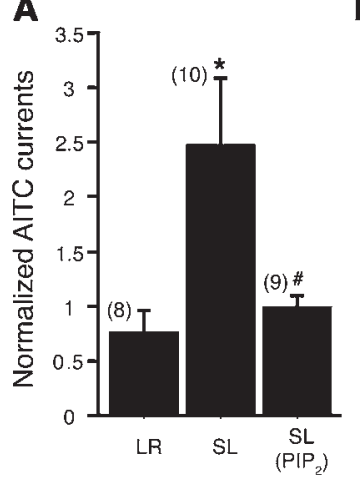

B Boiled $\mathrm{PIP}_{2} \mathrm{Ab} \quad \mathrm{PIP}_{2} \mathrm{Ab}$ in pipette (cont) in pipette

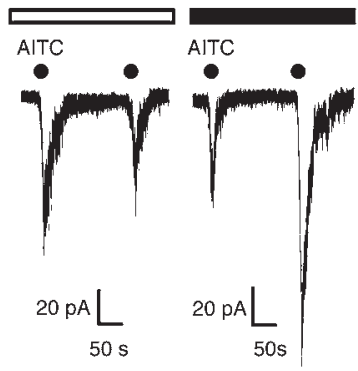

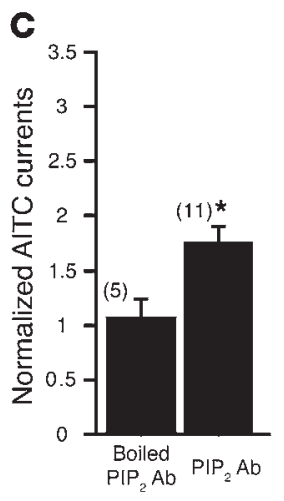

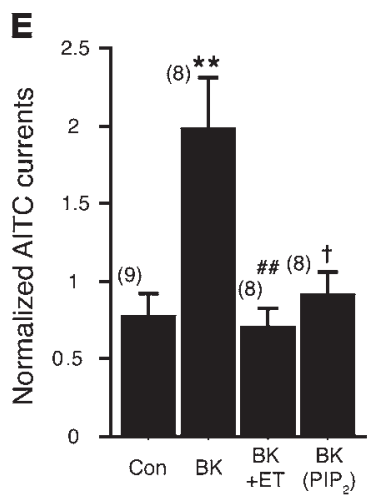

Figure 4

SL-NH2- or bradykinin-induced potentiation of AITC-activated currents in HEK cells transfected with hTRPA1 is mediated by PIP 2 hydrolysis. (A) A PIP 2 -dependent pathway is involved in the SL-NH2-induced potentiation of AITC-activated currents. AITC was reapplied 60 seconds after exposure to bath solution with SL-NH2 or LR-NH2. Currents were normalized to the values first induced by AITC application in the absence of SL-NH2 or LR-NH2. The potentiation by SL-NH2 was inhibited when a water-soluble $\mathrm{PIP}_{2}(10 \mu \mathrm{M})$ was added to pipette solution. ${ }^{\#} P<0.05$ versus SL-NH2; unpaired Student's $t$ test. ${ }^{*} P<0.05$ versus LR-NH2. (B) Representative traces show hTRPA1 currents produced by repeated applications of AITC $(100 \mu \mathrm{M})$ with the $\mathrm{PIP}_{2}$ antibody (right; 1:100) or boiled $\mathrm{PIP}_{2}$ antibody (boiled antibody was added to the pipette solution as a control) (left) in the pipette solution. A dialyzed mouse monoclonal $\mathrm{PIP}_{2}$ antibody (see Methods) was included in the pipette solution. $V_{\mathrm{h}}$ was $-60 \mathrm{mV}$. (C) The intracellular $\mathrm{PIP}_{2}$ antibody potentiated the AITC-evoked hTRPA1 response. Currents were normalized to values first induced by AITC application. ${ }^{*} P<0.05$ versus boiled $\mathrm{PIP}_{2}$ antibody; unpaired Student's $t$ test. (D) Intracellular application of polylysine $(3 \mu \mathrm{g} / \mathrm{ml})$, a PIP 2 scavenger, also potentiated AITC-activated currents in transfected HEK cells. ${ }^{*} P<0.05$ versus control; unpaired Student's $t$ test. (E) A PIP ${ }_{2}^{-}$ dependent pathway is involved in the bradykinin-induced potentiation of AITC activated currents in HEK cells transfected with B2R and hTRPA1. AITC was reapplied 60 seconds after exposure to bath solution with or without bradykinin. Currents were normalized to the values first induced by AITC application in the absence of bradykinin. The potentiation by bradykinin was inhibited when pretreated with the PLC inhibitor ET-18-OCH $(2 \mu \mathrm{M})$ for 120 seconds before bradykinin $(20 \mathrm{nM})$ application or a water-soluble $\mathrm{PIP}_{2}(10 \mu \mathrm{M})$ was added to pipette solution. ${ }^{*} P<0.005$ versus control; ${ }^{\dagger} P<0.05$ versus bradykinin (BK); ${ }^{\#} P<0.005$ versus BK; unpaired Student's $t$ test. Numbers in parentheses indicate cells tested.

and activated by the inflammatory peptide bradykinin, into HEK cells with TRPA1 and examined the effect of bradykinin on AITCevoked currents. Consistent with the observations obtained from PAR2, the B2R activation also potentiated AITC-evoked TRPA1 currents in a PLC/PIP ${ }_{2}$-dependent manner (Figure 4E).

PAR2 agonist sensitizes TRPA1 channel in DRG neurons in a PIP $2^{-}$ dependent manner. We next asked whether PAR2 activation would sensitize TRPA1 channels in sensory neurons. We performed voltage-clamp experiments in rat DRG neurons cultured without any proteinase treatment and examined the effects of SL-NH2 on the AITC-activated currents. Since an AITC-activated current in capsaicin-sensitive DRG neurons is certainly a TRPA1-mediated event (11), capsaicin $(1 \mu \mathrm{M})$ was applied to identify whether AITCactivated current was a TRPA1 current at the end of recording. In the voltage-clamp experiments, the AITC-activated $(300 \mu \mathrm{M})$ inward current generally underwent tachyphylaxis, giving smaller response on repeated applications (Figure 5A). However, after a 2 -minute pretreatment with $100 \mu \mathrm{M}$ SL-NH2, the same doses of AITC produced larger current responses than the first application of AITC, which was not pretreated with SL-NH2 (0.77 \pm 0.09 -fold change, $n=9$ for control; $1.51 \pm 0.13$-fold change, $n=7$ for SL-NH2, $P<0.005$ ) (Figure 5, B and C). These observations indicate that a PAR2 agonist potentiates TRPA1 activity in DRG neurons. PAR2 functionally interacted with TRPA1 in a PIP $_{2}$-dependent manner in the HEK293 cells with heterologous expression of TRPA1 in the present study. Since cell types differ in their membrane lipid composition, we then asked whether PAR2 activation would also sensitize TRPA1 channels with the same PIP $_{2}$-dependent mechanism in sensory neurons. In AITC-responsive neurons, as in the HEK293 cells expressing TRPA1 and PAR2, SL-NH2 failed to potentiate the AITC-activated currents when $\mathrm{PIP}_{2}$ was included in the pipette solution $\left(0.75 \pm 0.11\right.$-fold change, $n=6$ for $\mathrm{PIP}_{2}$, $P>0.05$ versus control, $P<0.05$ versus SL-NH2) (Figure $5 \mathrm{C}$ ). In contrast, intracellular application of polylysine, the $\mathrm{PIP}_{2}$ scavenger, potentiated the AITC-evoked currents $(1.31 \pm 0.19$-fold change, $n=7$ for polylysine, $P<0.05$ versus control) (Figure 5C).

To determine whether the potentiation of AITC-evoked current by PAR2 is due to sensitization of the channel or reversal of channel desensitization, we measured the AITC-evoked current density in the voltage-clamp recording with or without $\mathrm{PIP}_{2}$ (or polylysine) in pipette solution. We found that the AITC-evoked current density in rat DRG neurons was significantly smaller when recorded with pipette solution containing $\mathrm{PIP}_{2}$ than that with control pipette solution $(10.9 \pm 1.6 \mathrm{pA} / \mathrm{pF}, n=12$ for control, $4.5 \pm 1.5 \mathrm{pA} / \mathrm{pF}, n=6$ for $\left.\mathrm{PIP}_{2}, P<0.05\right)$. In contrast, the AITCevoked current density in rat DRG neurons was significantly larger when recorded with pipette solution containing polylysine than that with control pipette solution $(43.7 \pm 13.1 \mathrm{pA} / \mathrm{pF}$, $n=15$ for polylysine, $P<0.05)$. These results suggest that as well as in heterologous expression systems, endogenous $\mathrm{PIP}_{2}$ inhibits TRPA1 in DRG neurons, and this repression can be alleviated by agents that activate PLC. Thus, SL-NH2-induced potentiation of TRPA1 responsiveness indeed develops through sensitization of this channel but not reversal of the channel desensitization.

AITC- or cinnamaldebyde-evoked pain behavior is enhanced by PAR2 activation. Activation of TRPA1 by pungent natural products suggests a nociceptive role for TRPA1. To test whether PAR2 activation could enhance TRPA1-mediated pain behavior, we performed intraplantar injections with AITC and cinnamaldehyde after a subinflammatory-dose injection of SL-NH2 (32) and 

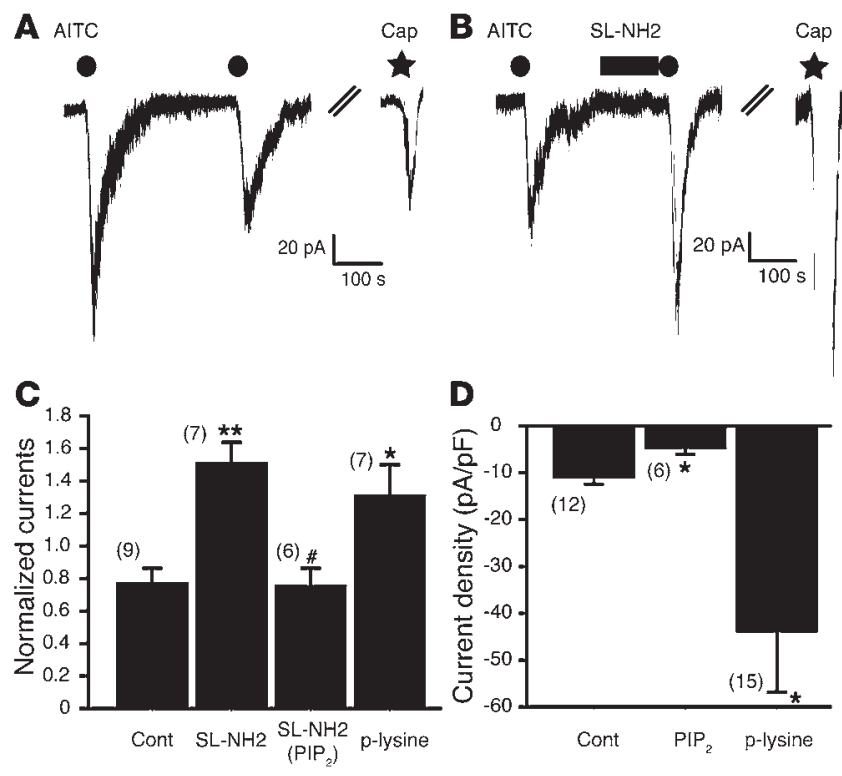

recorded nocifensive behaviors of the rats. Consistent with the previous studies $(3,12)$, both AITC and cinnamaldehyde injection induced a significant licking and lifting behavior of the injected hind paw during the 2- or 3-minute postinjection period, whereas such behaviors were not observed in vehicle-injected rats (data not shown). The subinflammatory dose injection of SL-NH2, as well as LR-NH2, did not cause acute nocifensive behaviors such as paw lifting, flinching, or licking. Ten minutes after these pretreatments, AITC or cinnamaldehyde was injected into the same area of the hind paw. We observed that pretreatment with SL-NH2 followed by intraplantar AITC or cinnamaldehyde injection induced a significant increase in the number of lifts and the duration of licks and flinches of the hind paw compared with pretreatment with LR-NH2 (Figure 6, A and B).

\section{Discussion}

Inflammatory pain is initiated by tissue damage/inflammation and is characterized by hypersensitivity, both at the site of damage and in adjacent tissue. In the context of inflammation, stimuli that normally would not produce pain do so (allodynia), whereas previously noxious stimuli evoke even greater pain responses (hyperalgesia). One mechanism underlying these phenomena is the modulation (sensitization) of ion channels, such as TRPV1, that detect noxious stimuli at the nociceptor terminal. Proteases such as trypsin or mast cell tryptase sensitize TRPV1 through a GPCR, PAR2 $(28,29)$. In the present study, we asked whether PAR2-mediated signaling could sensitize another TRP family member, TRPA1. We observed that PAR2 activation could potentiate TRPA1 activity, resulting in amplification of the pain sensation.

In recent years, a novel nociceptive pathway mediated by PAR2 has received much attention (25-27). Proteinases and PAR2 are known to cause hyperalgesia in response to both thermal and mechanical stimuli $(26,27)$. However, the underling mechanism of PAR2-mediated hyperalgesia has not been fully described, although involvement of the TRPV1 has been suggested (26, $28,29,33)$. We have previously shown that activation of PAR2 led to sensitization or potentiation of TRPV1 activity. However, SL-NH2-induced spinal Fos expression and pain behaviors were

\section{Figure 5}

SL-NH2 sensitizes TRPA1 channel in DRG neurons. (A and B) Representative traces of TRPA1-like currents by repeated AITC $(300 \mu \mathrm{M})$ application without $($ A) or with (B) SL-NH2 $(100 \mu \mathrm{M})$ treatment. AITC was reapplied 120 seconds after exposure to SL-NH2. Capsaicin (Cap; $1 \mu \mathrm{M})$ was applied to identify whether it was a TRPA1 current at the end of recording. (C) Effects of intracellular $\mathrm{PIP}_{2}$ on the PAR2-mediated sensitization of TRPA1. Currents were normalized to the currents evoked initially by AITC in the absence of the SL-NH2. In some experiments, a water-soluble $\mathrm{PIP}_{2}(10 \mu \mathrm{M})$ or polylysine was added to pipette solution. The potentiation by SL-NH2 was inhibited when the $\mathrm{PIP}_{2}$ was added to pipette solution. In contrast, intracellular application of polylysine (p-lysine; $3 \mu \mathrm{g} / \mathrm{ml}$ ), a PIP 2 scavenger, potentiated AITC-activated currents in DRG neurons. ${ }^{*} P<0.05,{ }^{* *} P<0.0005$, versus control; ${ }^{*} P<0.05$ versus SL-NH2; unpaired Student's $t$ test. (D) Effects of intracellular $\mathrm{PIP}_{2}$ on AITC-induced current density in DRG neurons. $\mathrm{PIP}_{2}$ or polylysine was added in the pipette solutions. ${ }^{*} P<0.05$ versus control; unpaired Student's $t$ test. Numbers in parentheses indicate cells tested.

not completely prevented in TRPV1-knockout mice in our previous study, suggesting the existence of other mechanisms downstream of PAR2 activation (28). The present results showed that TRPA1 is highly colocalized with PAR2 in primary sensory neurons and functionally interacts with PAR2. In addition to TRPV1, the interaction between PAR2 and TRPA1 might be an important mechanism underlying PAR2-mediated inflammatory pain.

In the present study, TRPA1 currents evoked by either of 2 different stimuli (AITC or cinnamaldehyde) were potentiated by PAR2 activation. The lack of potentiating effects of PAR2 agonists in cells treated with PLC inhibitors, and the similar potentiating effect of the PLC activator, indicate that a PLC-dependent pathway is predominantly involved in PAR2-mediated TRPA1 sensitization. PLC activity plays a major role in the regulation and activation of many TRP channels (30). One of the consequences of PLC activation is the breakdown of $\mathrm{PIP}_{2}$ into DAG and inositol triphosphate (IP3). IP3 then releases calcium from internal stores, while DAG activates PKC and can also be converted to AA by DAG lipase. Activation of PAR2 results in intracellular $\mathrm{Ca}^{2+}$ mobilization (25), and a recent study indicated that intracellular calcium may directly activate TRPA1 (8). However, it is not a likely mechanism for the AITC-evoked current increase observed in our experiments, as cytosolic free calcium was tightly chelated with the 5-mM EGTA included in the pipette solution. A recent study using calcium imaging indicated that long exposure to OAG (>180 s) might activate TRPA1 through its metabolites such as AA (3). However, we can note that SL-NH2- or PLC activator-induced potentiation of the AITC-activated currents occurred rapidly ( $\leq 90 \mathrm{~s}$ ) and that a 90 -second exposure to OAG in our experiment did not cause an increase in the AITC-activated current. Therefore, DAG and its metabolites are not likely involved in PLC-mediated potentiation under the present experimental paradigm. Indeed, long application of OAG may activate the TRPA1 channel (3). In any case, the sensitization mechanisms and the activation mechanism of TRPA1 should be considered separately.

In contrast to the TRPV1-regulating mechanism of PAR2, we found that the PKC inhibitor did not prevent the potentiation of AITC-evoked current by PAR2 activation. Moreover, the PKC activator did not potentiate the AITC-evoked current. These observations suggest that the downstream products of PLC activation do not contribute to sensitization of TRPA1. Thus, the remaining possible mechanism is the consequence of membrane $\mathrm{PIP}_{2}$ hydrolysis 

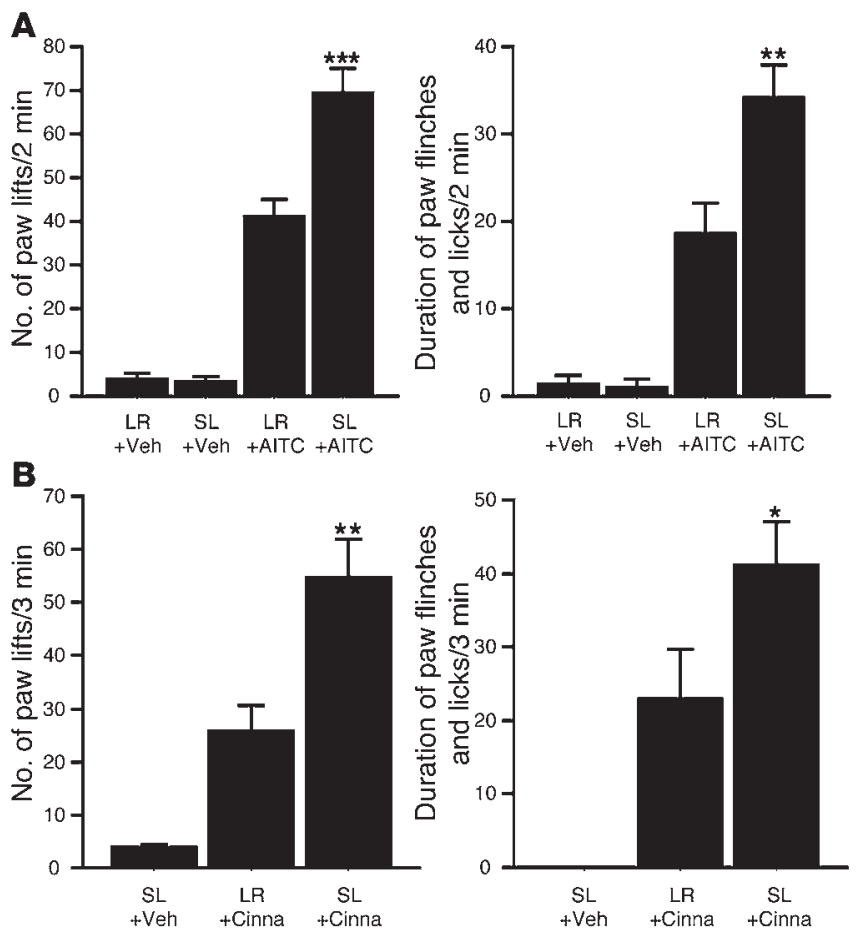

by PLC activation. The $\mathrm{PIP}_{2}$ has been shown to regulate the activity of a variety of ion channels (31), including inwardly rectifying $\mathrm{K}^{+}$(Kir) channels and the channels of the TRP family (31, 34-38). TRPM7 (39), TRPM5 (40), and TRPM8 (41) were activated by PIP whereas TRPL (42) and TRPV1 (43) were inhibited by PIP 2 . Indeed, in this study we observed that intracellular application of $\mathrm{PIP}_{2}$ completely abolished the potentiation of AITC-evoked current by PAR2 activation. A similar mechanism was also found in another GPCR (B2R) activation-induced potentiation of TRPA1; thus, we conclude that the PLC/PIP 2 -mediated sensitization mechanism of TRPA1 is a common property in the modulation of TRPA1 channel by GPCR. Evidence that antibody against $\mathrm{PIP}_{2}$ or polylysine (the scavenger of $\mathrm{PIP}_{2}$ ) in the pipette solution potentiated AITC-evoked currents similarly to PAR2 or B2R activation further supported this conclusion.

If PAR 2 activation can sensitize TRPA1, pain sensation that was caused through the TRPA1 channel may also be potentiated by the PAR2 activation. Topical application of agents, such as AITC or cinnamaldehyde, has been reported to excite sensory nerve fibers, thereby producing acute pain $(3,4,12)$. In the behavior studies, we found that pretreatment of SL-NH2 in a subinflammatory dose led to an increase in AITC- or cinnamaldehyde-evoked nocifensive behavior in rats. These results indicate that PAR2 activation indeed increased TRPA1 activity, not only at the cellular level but also at the behavioral level.

Tryptase and trypsin, the physiological agonists of PAR2, may be released from peripheral tissue and visceral organs in inflammatory diseases. Our results represent what we believe to be a novel mechanism through which trypsin or tryptase released in response to tissue inflammation might trigger the sensation of pain through PAR2 activation. Although proteinase inhibitors and PAR2 antagonists seem to be effective for antiinflammation and antinociception, the absence of selective PAR2 antagonists and proteinase inhibitors makes it difficult to perform experimental studies in animals or humans. PAR2 is expressed in a wide variety

\section{Figure 6}

TRPA1 activation-induced nocifensive behaviors that were potentiated by SL-NH2. Animals were pretreated subcutaneously with SL-NH2 or LR-NH2 10 minutes before AITC or cinnamaldehyde injection. (A) Pain response to subcutaneous AITC. AITC ( $3 \%$ in $50 \mu$ l liquid paraffin) was injected into the left hind footpad, and the number of paw lifts (left) and duration of paw flinches and licks (right) were measured in the first 2 minutes after injection of AITC. Injection of SL-NH2 or LR-NH2 with vehicle did not cause notable paw lifts or flinches $(L R+V e h ; S L$ + Veh). The AITC injections caused significant nocifensive behaviors after SL-NH2 or LR-NH2 pretreatment; however, preinjection of $\mathrm{SL}-\mathrm{NH} 2(10 \mu \mathrm{g}$ in $50 \mu \mathrm{l}$ saline) significantly increased the number of paw lifts and the duration of paw flinches and licks, compared with preinjection of LR-NH2 (10 $\mu \mathrm{g}$ in $50 \mu \mathrm{l}$ saline). ${ }^{\star * \star} P<0.0001,{ }^{\star \star} P<0.001$ versus $L R+$ AITC. (B) The pain response to subcutaneous cinnamaldehyde (Cinna). Cinnamaldehyde (100\%, $50 \mu \mathrm{l})$ was injected into the left hind footpad, and the number of paw lifts (left) and duration of paw flinches and licks (right) were measured in the first 3-minute period. Note that SL-NH2 preinjection significantly increased the number of paw lifts and the duration of paw flinches and licks compared with LR-NH2 preinjection. ${ }^{*} P<0.05,{ }^{* \star} P<0.001$ versus $\mathrm{LR}+$ Cinna. $n=6$ rats in each experiment. ANOVA followed by Fisher's protected least square difference tests were used in the statistical analysis.

of tissues, including lung, liver, kidney, and the gastrointestinal tract, where it might be involved in physiological and pathophysiological processes, including mucosal protection and repair (32, 44-47). Therefore, it is possible that blockade of PAR2 could be associated with adverse effects in these tissues. Because our present data demonstrated that PAR2 activation could functionally sensitize TRPA1, which has been reported to be involved in cold and mechanical sensation and inflammatory actions of environmental irritants and proalgesic agents, compounds acting on TRPA1 or interfering with the interaction between TRPA1 and PAR2 may be useful in the treatment of inflammatory pain.

\section{Methods}

Generation of TRPA1 antisera. To raise antibodies against TRPA1 proteins, rabbits were injected with the following peptide: RFKKERLEQMHSKWNF coupled via the cysteine to keyhole limpet hemocyanin (KLH) (48). The peptide corresponds to a sequence present in the constant region of the rat or mouse proteins (residues 1092-1107 in NP_997491 for rat, residues 1092-1107 in NP_808449 for mouse). The KLH-linked TRPA1 peptide was injected into rabbits using standard procedures for antiserum production by Peptide Institute Inc. For affinity purification, the peptide was coupled to an affinity column made of aminoalkyl agarose (Bio-Rad), and the antibody was purified following the manufacturer's protocols.

Western blotting. Rats were deeply anesthetized with diethyl ether and killed by decapitation. In accordance with the procedure of our previous report (49), tissue samples from the DRGs were removed and then lysed by homogenizing in $200 \mu \mathrm{l} 20 \mathrm{mM}$ Tris-HCI buffer, $\mathrm{pH}$ 8.0, containing $1 \%$ NP40, $150 \mathrm{mM} \mathrm{NaCl}, 1 \mathrm{mM}$ EDTA, 10\% glycerol, 0.1\% $\beta$-mercaptoethanol, $0.5 \mathrm{mM}$ dithiothreitol, and a mixture of proteinase inhibitors. The supernatant containing $15 \mu \mathrm{g}$ of protein was electrophoresed in a $10-20 \%$ SDS-polyacrylamide gel (Bio-Rad) and blotted onto Hybond P membranes (Amersham) using Multiphore II (Amersham) for 45 minutes. Membranes were incubated with $1 \%$ BSA in Tris buffer saline containing Tween-20 (TBST; $10 \mathrm{mM}$ Tris-HCl, $\mathrm{pH} 8.0,150 \mathrm{mM} \mathrm{NaCl}$, and $0.2 \%$ Tween-20) for at least 10 minutes at room temperature and incubated with the anti-TRPA1 antibody (1:500) at $4^{\circ} \mathrm{C}$ overnight. The membranes were then incubated with an alkaline phosphatase-conjugated goat anti-rabbit $\operatorname{IgG}(1: 5,000$; Jackson ImmunoResearch Laboratories Inc.) 2 hours at room tempera- 
ture. Finally, membranes were washed several times with TBST to remove unbound secondary antibodies and visualized using a BCIP-NBT Solution Kit for alkaline phosphatase stain (Nacalai Tesque Inc.). In some experiment, HEK293 cells expressing mouse TRPA1 were used for the Western blotting by the same protocol.

Immunohistochemistry. Adult male Sprague-Dawley rats (220-250 g; Japan Animals) were perfused transcardially with $1 \%$ paraformaldehyde in $0.1 \mathrm{M}$ phosphate buffer followed by $4 \%$ paraformaldehyde in $0.1 \mathrm{M}$ phosphate buffer ( $\mathrm{pH}$ 7.4). The L4-5 DRGs were removed and processed for TRPA1 immunohistochemistry as described in our previous study (49). The polyclonal primary antibody for TRPA1 at 1:2,000 was used for DAB staining. For double immunofluorescence, tyramide signal amplification (TSA; NEN) fluorescence procedures (50) were used for TRPA1 $(1: 10,000)$ staining. Then the TRPA1 antibody was combined with 1 of the following antibodies: monoclonal anti-NF200 (1:400; Sigma-Aldrich), rabbit polyclonal TRPV1 antibody (1:1,000; Oncogene Research Products), and mouse monoclonal antibody against PAR2 (1:100; Santa Cruz Biotechnology Inc.) as described in our previous study (49). When 2 primary antisera raised in rabbit were combined, nonspecific double labeling was not observed. The lack of cross-reactivity is thought to be attributable to the fact that the TSA procedure allows the first series primary antibody to be used at a dilution that is too high to be detected by the second reagent set (50). Our data support this explanation. In control single labeling using indirect labeled immunofluorescence, we were unable to visualize the TRPA1 antiserum at the dilutions used for the TSA procedure.

Mammalian cell culture. HEK293 cells were maintained in DMEM (supplemented with 10\% FBS, 2 mM Glutamax (Invitrogen), penicillin, and streptomycin) and transfected with $1 \mu \mathrm{g}$ of human or mouse TRPA1 (hTRPA1 and mTRPA1, respectively) cDNA, or mouse bradykinin receptor (mB2R) cDNA using Lipofectamine 2000 (Invitrogen). The TRPA1 cDNAs and mB2R cDNA were a generous gift from Ardem Patapoutian (The Scripps Research Institute, La Jolla, California, USA) and Haruhiro Higashida (Kanazawa University Graduate School of Medicine, Kanazawa, Japan), respectively. To identify transfected cells, an enhanced green fluorescence protein reporter plasmid was also transfected at $0.1 \mu \mathrm{g}$. For primary culture of DRG neurons, DRGs were collected from the adult Sprague-Dawley rats (100-200 g) under sterile conditions, and placed in ice-cold Earle's balanced salt solution (EBSS; Sigma-Aldrich). Adhering fat and connective tissue were removed, and each DRG was placed immediately in medium consisting of $2 \mathrm{ml}$ of EBSS containing $1.25 \mathrm{mg} / \mathrm{ml}$ of collagenase $\mathrm{P}$ (Sigma-Aldrich) and kept at $37^{\circ} \mathrm{C}$ for 60 minutes with occasional agitation. After dissociation of the cell ganglia, this cell suspension was centrifuged for 5 minutes at $650 \mathrm{~g}$, and the cell pellet was resuspended in EBSS supplemented with $10 \% \mathrm{FBS}, 2 \mathrm{mM}$ Glutamax, penicillin, and streptomycin. Recombinant rat nerve growth factor $(100 \mathrm{ng} / \mathrm{ml}$; R\&D Systems) was added to the medium.

Electrophysiology. Whole-cell patch-clamp recordings were carried out at 2 days after transfection of hTRPA1 cDNA into HEK293 cells or at 1 day after dissociation of the DRG neurons. Voltage-clamp experiments were performed at a holding potential of $-60 \mathrm{mV}$, and recordings were sampled at $5 \mathrm{kHz}$ and filtered at $2 \mathrm{kHz}$. The current-voltage relationships were determined using a 340-ms voltage ramp from $-120 \mathrm{mV}$ to $+100 \mathrm{mV}$. Current density $(\mathrm{pA} / \mathrm{pF})$ or normalized current (currents were normalized to the currents evoked initially by agonist) were measured. A normalized current was obtained just in case the initial current by agonist was smaller than $400 \mathrm{pA}$ to prevent a large current-induced strong desensitization. The current magnitude was quantified by peak current amplitude in all experiments. Standard bath solution contained $140 \mathrm{mM} \mathrm{NaCl}, 5 \mathrm{mM} \mathrm{KCl}, 2 \mathrm{mM} \mathrm{MgCl}_{2}$, $2 \mathrm{mM} \mathrm{CaCl}_{2}, 10 \mathrm{mM}$ HEPES, and $10 \mathrm{mM}$ glucose, $\mathrm{pH} 7.4$ (adjusted with $\mathrm{NaOH}$ ). The pipette solution contained $135 \mathrm{mM}$ potassium gluconate, $5 \mathrm{mM} \mathrm{KCl}, 2 \mathrm{mM} \mathrm{MgCl}_{2}, 0.5 \mathrm{mM} \mathrm{CaCl}_{2}, 5 \mathrm{mM} \mathrm{MgATP}, 5 \mathrm{mM}$ EGTA, and 5 mM HEPES, pH 7.2 (adjusted with Tris-base). In some experiments, a PAR2 agonist, SL-NH2 (10-100 $\mu \mathrm{M}$; Peptide Institute Inc.) or its reversed inactive form, LR-NH2 (Peptide Institute Inc.) was contained in bath solutions. The $100-\mu \mathrm{M}$ dose SL-NH2 has been used in many studies to activate PAR2 receptor $(27,28,51)$. AITC (30 or $100 \mu \mathrm{M}$; Nacalai Tesque Inc.) or cinnamaldehyde (500 $\mu \mathrm{M}$; Sigma-Aldrich) were used to stimulate TRPA1. In some experiments, a mouse monoclonal $\mathrm{PIP}_{2}$ antibody (1:100 [Abcam], dialyzed with Slid-A-Lyzer 10K MWCO Dialysis Cassettes; Pierce), a watersoluble $\mathrm{PIP}_{2}(10 \mu \mathrm{M}$; Calbiochem), or poly-L-lysine (MW 30,000-70,000 Da, $3 \mu \mathrm{g} / \mathrm{ml}$; Sigma-Aldrich) was included in the pipette solution. All patchclamp experiments were performed at room temperature $\left(\sim 25^{\circ} \mathrm{C}\right)$. The solutions containing drugs were applied to the chamber $(180 \mu \mathrm{l})$ by a gravity system at a flow rate of 3-4 $\mathrm{ml} / \mathrm{min}$.

Behavioral studies. Fifty-four adult male Sprague-Dawley rats (200-250 g) were used for the behavioral analyses. After adaptation, SL-NH2, the PAR2 agonist, at $10 \mu \mathrm{g}$ in $50 \mu \mathrm{l}$ saline or LR-NH2, the reversed inactive form of SL-NH2, at $10 \mu \mathrm{g}$ in $50 \mu \mathrm{l}$ saline was injected intraplantarly into the left hind paw of each rat. Ten minutes after injection, rats received intradermal injection of $50 \mu \mathrm{l}$ of AITC ( $3 \%$ in liquid paraffin; Wako) or $100 \%$ cinnamaldehyde to the plantar surface of the left hind paw. Liquid paraffin was used as a vehicle control in these experiments. The rats were placed in a wire mesh cage immediately after the injection, and the numbers of hind paw lifts and the duration of hind paw flinches and licks during the initial 2-minute (AITC) or 3-minute period (cinnamaldehyde) were measured. All procedures involving the care and use of animals were approved by the Hyogo College of Medicine Committee on Animal Research and were carried out in accordance with the NIH guidelines for the care and use of laboratory animals.

Statistics. All results are expressed as mean \pm SEM. An unpaired 1-tailed Student's $t$ test was used to compare the electrophysiological data between the 2 groups. ANOVA followed by Fisher's protected least square difference tests were applied to the behavioral data. A difference was accepted as significant if $P$ was less than 0.05 .

\section{Acknowledgments}

This work was supported in part by Grants-in-Aid for Scientific Research and the Open Research Center grant, Hyogo College of Medicine, both from the Japanese Ministry of Education, Culture, Sports, Science, and Technology. We gratefully acknowledge technical assistance from Noriko Kusumoto. We thank D.A. Thomas for correcting the English usage in this manuscript.

Received for publication November 14, 2006, and accepted in revised form April 10, 2007.

Address correspondence to: Koichi Noguchi, Department of Anatomy and Neuroscience, Hyogo College of Medicine, 1-1 Mukogawa-cho, Nishinomiya, Hyogo 663-8501, Japan. Phone: 0798-456415; Fax: 0798-45-6417; E-mail: noguchi@hyo-med.ac.jp.
1. Jaquemar, D., Schenker, T., and Trueb, B. 1999. An ankyrin-like protein with transmembrane domains is specifically lost after oncogenic transformation of human fibroblasts. J. Biol. Chem. 274:7325-7333.

2. Story, G.M., et al. 2003. ANKTM1, a TRP-like channel expressed in nociceptive neurons, is activated by cold temperatures. Cell. 112:819-829.

3. Bandell, M., et al. 2004. Noxious cold ion channel TRPA 1 is activated by pungent compounds and bradykinin. Neuron. 41:849-857.

4. Jordt, S.E., et al. 2004. Mustard oils and cannabinoids excite sensory nerve fibres through the TRP channel ANKTM1. Nature. 427:260-265.

5. Macpherson, L.J., et al. 2005. The pungency of garlic: activation of TRPA1 and TRPV1 in response to allicin. Curr. Biol. 15:929-934.

6. Hinman, A., Chuang, H.H., Bautista, D.M., and Julius, D. 2006. TRP channel activation by reversible 
covalent modification. Proc. Natl. Acad. Sci. U. S. A. 103:19564-19568.

7. Macpherson, L.J., et al. 2007. Noxious compounds activate TRPA1 ion channels through covalent modification of cysteines. Nature. 445:541-545.

8. Zurborg, S., Yurgionas, B., Jira, J.A., Caspani, O., and Heppenstall, P.A. 2007. Direct activation of the ion channel TRPA1 by $\mathrm{Ca}(2+)$. Nat. Neurosci. 10:277-279.

9. Nagata, K., Duggan, A., Kumar, G., and GarciaAnoveros, J. 2005. Nociceptor and hair cell transducer properties of TRPA1, a channel for pain and hearing. J. Neurosci. 25:4052-4061.

10. Kobayashi, K., et al. 2005. Distinct expression of TRPM8, TRPA1, and TRPV1 mRNAs in rat primary afferent neurons with adelta/c-fibers and colocalization with trk receptors. J. Comp. Neurol. 493:596-606.

11. Kwan, K.Y., et al. 2006. TRPA1 contributes to cold, mechanical, and chemical nociception but is not essential for hair-cell transduction. Neuron. 50:277-289.

12. Bautista, D.M., et al. 2006. TRPA1 mediates the inflammatory actions of environmental irritants and proalgesic agents. Cell. 124:1269-1282.

13. Vu, T.K., Hung, D.T., Wheaton, V.I., and Coughlin, S.R. 1991. Molecular cloning of a functional thrombin receptor reveals a novel proteolytic mechanism of receptor activation. Cell. 64:1057-1068.

14. Ishihara, H., et al. 1997. Protease-activated receptor 3 is a second thrombin receptor in humans. Nature. 386:502-506.

15. Kahn, M.L., et al. 1998. A dual thrombin receptor system for platelet activation. Nature. 394:690-694.

16. Nystedt, S., Emilsson, K., Wahlestedt, C., and Sundelin, J. 1994. Molecular cloning of a potential proteinase activated receptor. Proc. Natl. Acad. Sci.U.S. A. 91:9208-9212.

17. Molino, M., et al. 1997. Interactions of mast cell tryptase with thrombin receptors and PAR2.J. Biol. Chem. 272:4043-4049.

18. Dery, O., Corvera, C.U., Steinhoff, M., and Bunnett, N.W. 1998. Proteinase-activated receptors: novel mechanisms of signaling by serine proteases. Am. J. Physiol. 274:C1429-C1452.

19. Hollenberg, M.D. 1999. Protease-activated receptors: PAR4 and counting: how long is the course? Trends Pharmacol. Sci. 20:271-273.

20. Hollenberg, M.D., and Compton, S.J. 2002. International Union of Pharmacology. XXVIII. Proteinaseactivated receptors. Pharmacol. Rev. 54:203-217.

21. Saifeddine, M., al-Ani, B., Cheng, C.H., Wang, L., and Hollenberg, M.D. 1996. Rat proteinase-activated receptor-2 (PAR2): cDNA sequence and activity of receptor-derived peptides in gastric and vascular tissue. Br. J. Pharmacol. 118:521-530.

22. Vergnolle, N., Hollenberg, M.D., Sharkey, K.A., and Wallace, J.L. 1999. Characterization of the inflam- matory response to proteinase-activated receptor-2 (PAR2)-activating peptides in the rat paw. $\mathrm{Br}$. J. Pharmacol. 127:1083-1090.

23. Vergnolle, N., Wallace, J.L., Bunnett, N.W., and Hollenberg, M.D. 2001. Protease-activated receptors in inflammation, neuronal signaling and pain. Trends Pharmacol. Sci. 22:146-152.

24. Seeliger, S., et al. 2003. Proinflammatory role of proteinase-activated receptor- 2 in humans and mice during cutaneous inflammation in vivo. FASEB J. 17:1871-1885.

25. Steinhoff, M., et al. 2000. Agonists of proteinaseactivated receptor 2 induce inflammation by a neurogenic mechanism. Nat. Med. 6:151-158.

26. Hoogerwerf, W.A., et al. 2001. The proteinase-activated receptor 2 is involved in nociception. J. Neurosci. 21:9036-9042.

27. Vergnolle, N., et al. 2001. Proteinase-activated receptor- 2 and hyperalgesia: a novel pain pathway. Nat. Med. 7:821-826.

28. Dai, Y., et al. 2004. Proteinase-activated receptor 2mediated potentiation of transient receptor potential vanilloid subfamily 1 activity reveals a mechanism for proteinase-induced inflammatory pain. J. Neurosci. 24:4293-4299.

29. Amadesi, S., et al. 2004. Protease-activated receptor 2 sensitizes the capsaicin receptor transient receptor potential vanilloid receptor 1 to induce hyperalgesia. J. Neurosci. 24:4300-4312.

30. Minke, B. 2001. The TRP channel and phospholipase C-mediated signaling. Cell. Mol. Neurobiol. 21:629-643.

31. Hilgemann, D.W., Feng, S., and Nasuhoglu, C. 2001. The complex and intriguing lives of PIP2 with ion channels and transporters. Sci. STKE. 2001:RE19.

32. Fiorucci, S., et al. 2001. Proteinase-activated receptor 2 is an anti-inflammatory signal for colonic lamina propria lymphocytes in a mouse model of colitis. Proc. Natl. Acad. Sci. U. S. A. 98:13936-13941.

33. McLean, P.G., Aston, D., Sarkar, D., and Ahluwalia, A. 2002. Protease-activated receptor-2 activation causes EDHF-like coronary vasodilation: selective preservation in ischemia/reperfusion injury: involvement of lipoxygenase products, VR1 receptors, and C-fibers. Circ. Res. 90:465-472.

34. Huang, C.L., Feng, S., and Hilgemann, D.W. 1998. Direct activation of inward rectifier potassium channels by PIP2 and its stabilization by Gbetagamma. Nature. 391:803-806.

35. Sui, J.L., Petit-Jacques, J., and Logothetis, D.E. 1998. Activation of the atrial KACh channel by the betagamma subunits of $G$ proteins or intracellular $\mathrm{Na}+$ ions depends on the presence of phosphatidylinositol phosphates. Proc. Natl. Acad. Sci. U. S. A. 95:1307-1312.

36. Zhang, H., He, C., Yan, X., Mirshahi, T., and Logo- thetis, D.E. 1999. Activation of inwardly rectifying $\mathrm{K}+$ channels by distinct PtdIns(4,5)P2 interactions. Nat. Cell Biol. 1:183-188.

37. Du, X., et al. 2004. Characteristic interactions with phosphatidylinositol 4,5-bisphosphate determine regulation of kir channels by diverse modulators. J. Biol. Chem. 279:37271-37281.

38. Hardie, R.C. 2003. Regulation of TRP channels via lipid second messengers. Annu. Rev. Physiol. 65:735-759.

39. Liu, D., and Liman, E.R. 2003. Intracellular Ca2+ and the phospholipid PIP2 regulate the taste transduction ion channel TRPM5. Proc. Natl. Acad. Sci. U. S. A. 100:15160-15165.

40. Runnels, L.W., Yue, L., and Clapham, D.E. 2002. The TRPM7 channel is inactivated by $\mathrm{PIP}(2)$ hydrolysis. Nat. Cell Biol. 4:329-336.

41. Liu, B., and Qin, F. 2005. Functional control of coldand menthol-sensitive TRPM8 ion channels by phosphatidylinositol 4,5-bisphosphate. J. Neurosci. 25:1674-1681.

42. Estacion, M., Sinkins, W.G., and Schilling, W.P. 2001. Regulation of Drosophila transient receptor potential-like ( $\operatorname{Tr} \mathrm{L}$ ) channels by phospholipase Cdependent mechanisms. J. Physiol. 530:1-19.

43. Chuang, H.H., et al. 2001. Bradykinin and nerve growth factor release the capsaicin receptor from PtdIns(4,5)P2-mediated inhibition. Nature. 411:957-962.

44. Lindner, J.R., et al. 2000. Delayed onset of inflammation in protease-activated receptor-2-deficient mice. J. Immunol. 165:6504-6510.

45. Reinshagen, M., et al. 2000. Protective role of neurotrophins in experimental inflammation of the rat gut. Gastroenterology. 119:368-376.

46. Kawabata, A., et al. 2001. The protease-activated receptor-2 agonist induces gastric mucus secretion and mucosal cytoprotection. J. Clin. Invest. 107:1443-1450.

47. Cocks, T.M., et al. 1999. A protective role for protease-activated receptors in the airways. Nature. 398:156-160.

48. Harlow, E., and Lane, D. 1988. Antibodies: a laboratory manual. Cold Spring Harbor Laboratory Press. Cold Spring Harbor, New York, USA. 726 pp.

49. Dai, Y., et al. 2002. Phosphorylation of extracellular signal-regulated kinase in primary afferent neurons by noxious stimuli and its involvement in peripheral sensitization. J. Neurosci. 22:7737-7745.

50. Michael, G.J., et al. 1997. Nerve growth factor treatment increases brain-derived neurotrophic factor selectively in TrkA-expressing dorsal root ganglion cells and in their central terminations within the spinal cord. J. Neurosci. 17:8476-8490.

51. Steinhoff, M., et al. 2003. Proteinase-activated receptor-2 mediates itch: a novel pathway for pruritus in human skin. J. Neurosci. 23:6176-6180. 